\title{
THE SCHUR GROUP CONJECTURE FOR THE RING OF INTEGERS OF A NUMBER FIELD
}

\author{
PETER NELIS
}

(Communicated by Maurice Auslander)

\begin{abstract}
If $R$ is the ring of $\mathbb{S}$ integers of a subcyclotomic number field $K$, then the Schur group conjecture asserts that the Schur group of $R$ equals the intersection of the Brauer group of $R$ and the Schur group of $K$. We prove this assertion in case $\mathbb{S}$ is the set of all Archimedian primes, i.e. when $R$ is the ring of integers of $K$.
\end{abstract}

\section{INTRODUCTION}

If $R$ is any commutative ring, then a Schur algebra over $R$ is an Azumaya algebra over $R$, which is the epimorphic image of a group ring $R G$, where $G$ is a finite group. In case $R$ is a field, this means that $A$ appears as a component of some group algebra $R G$. One now defines the Schur group $\mathrm{S}(R)$ of $R$ as the subgroup of the Brauer group consisting of the classes, up to Brauer equivalence, of Schur algebras over $R$. If $R$ is a Dedekind domain, then $\operatorname{Br}(R)$ is a subgroup of $\operatorname{Br}(K)$ and we clearly have

$$
\mathrm{S}(R) \subseteq \mathrm{Br}(R) \cap \mathrm{S}(K) .
$$

In [16] Riehm states a conjecture of E. B. Williams which asserts that equality holds whenever $R$ is the ring of $\mathbb{S}$ integers of any subcyclotomic number field, where $\mathbb{S}$ is any set of places. In [17] he then proves the equality for the case of cyclotomic number rings. In case $\mathbb{S}$ is the set of all finite places, the Schur group conjecture was only known when the right-hand side is trivial, except for a few exceptions. The fact that no analogue of the Brauer Witt theorem for fields has been obtained for the integral case, seriously hampers the calculation of these Schur groups. In this note, we prove the Schur group conjecture when $R$ is the ring of integers of any number field, i.e. when $\mathbb{S}$ is the set of all finite places. First, we prove that the Schur group conjecture may be reduced to the quadratic case. We then pay attention to the particular situation $K=\mathbb{Q}(\sqrt{p})$, $p$ prime. A cyclic crossed product order over $\mathbb{Z}[(1+\sqrt{p}) / 2]$ may be obtained

Received by the editors June 19, 1990 and, in revised form, September 11, 1990.

1980 Mathematics Subject Classification (1985 Revision). Primary 20C10, 20C25, 13A20; Secondary $16 \mathrm{~A} 16,16 \mathrm{~A} 26$.

Key words and phrases. Group rings, Azumaya algebras, the integral Brauer group, the Schur group, linear and modular representations.

Sponsored by the Belgian National Science Foundation NFWO. 
by adjoining a $p$ th root of unity and defining a 2-cocycle that takes values in the roots of unity. This algebra is the epimorphic image of a group ring, but it is not Azumaya. However, one may extend the representation to a larger group, viz. $\mathrm{Sl}_{2}\left(\mathbb{F}_{p}\right)$, where $\mathbb{F}_{p}$ denotes the Galois field of order $p$. The epimorphic image of this group is then Azumaya. We apply this to prove the Schur group conjecture for primes $p, p \equiv 1 \bmod 4$. Starting from these bricks and a weak multiplicative law for the values of $\mathbb{N}$ for which the Schur group conjecture is true, an induction process may be set up to prove the theorem in general. The calculation of the Schur groups of the ring of integers of a number field then ensues immediately.

\section{THE SCHUR GROUP OF NUMBER RINGS}

Throughout, $K$ is a number field, $R$ its ring of integers or a localization of this ring, $k$ is the quotient field of $R$ modulo some prime ideal $P, \operatorname{Br}(R)$ is the Brauer group of $R, G$ is a finite group, $\varepsilon_{n}$ is an $n$th root of unity and unadorned tensor product is over $R$.

Definition 1.1. An Azumaya algebra $A$ over $R$ is a Schur algebra if and only if there exists a finite group $G$ and an $R$ algebra epimorphism from the group algebra $R G$ onto $A$.

Example 1.2. The Schur algebras over $\mathbb{Z}$ are precisely the matrix rings $M_{n}(\mathbb{Z})$, where $n=1$ or $8 \mid n$. We will sketch the proof of this result, for details the reader is referred to [12]. The case $n=1$ is clearly trivial, so assume $n>1$. If $G$ is a finite subgroup of $\mathrm{Gl}_{n}(\mathbb{Z})$ such that the $\mathbb{Z}$ module generated by $G$ is $M_{n}(\mathbb{Z})$, then $G$ can be viewed as the subgroup of the automorphism group of an even unimodular quadratic form. This was proved independently by Thompson in [20] and by the author in [12]. If $\Gamma_{8}$ denotes the unique unimodular, even quadratic form of dimension 8 , then one can prove that the automorphism group of $k$ copies of $\Gamma_{8}$ spans $M_{8 k}(\mathbb{Z})$ as a $\mathbb{Z}$ module.

Definition 1.3. $\mathrm{S}(R)=\{[A] \in \operatorname{Br}(R), A$ is a Schur algebra $\}$ is called the Schur group of $R$.

It is easy to prove that $\mathrm{S}(R)$ is indeed a group (cf. [4] or [11]). For a generalization of this concept to twisted group rings as well as for general theorems concerning these Schur groups, the reader is referred to [11]. If $A$ is an Azumaya algebra over $R$, then $A \otimes K$ is a central simple algebra. This yields a map

$$
\operatorname{Br}(R) \rightarrow \operatorname{Br}(K):[A] \mapsto[K \otimes A] .
$$

Since $R$ is a Dedekind domain, this map is an injection (cf. [13, Theorem 6.19, p. 67]). We will therefore view $\operatorname{Br}(R)$ as a subgroup of $\operatorname{Br}(K)$. If furthermore $\pi: R G \rightarrow A$ is an epimorphism, then so is $\pi: K G \rightarrow K \otimes A$. This shows that

$$
\mathrm{S}(R) \subseteq \operatorname{Br}(R) \cap \mathrm{S}(K) .
$$

The Schur group conjecture asserts that equality holds when $R$ is the ring of $\mathbb{S}$ integers of a subcyclotomic number field. This has been proven in [17] for the case $R=\mathbb{Z}\left[\varepsilon_{n}\right], n$ arbitrary and for any cofinite set of places $\mathbb{S}$ not containing the infinite ones. We recall that the ring of $\mathbb{S}$ integers is then defined as the set of elements of $K$ that has a positive valuation at every place of $\mathbb{S}$. 
If $\mathbb{S}$ is the set of all finite places, then we call $R_{\mathbb{S}}$ the number ring of $K$. This ring is then the integral closure of $\mathbb{Z}$ in $K$. We will now proceed to prove that equality holds when $K$ is an arbitrary subcyclotomic number field and $R$ its number ring. If the Schur group conjecture holds, then this allows an effective computation of the Schur group of the ring of $\mathbb{S}$ integers for any number field $K$ and any finite set of places $\mathbb{S}$. Indeed, let $K_{c}$ be the maximal subcyclotomic field contained in $K$ and let $R_{c}=R \cap K_{c}$. If $A_{c}$ is a Schur algebra over $R_{c}$, then $R \otimes_{R_{c}} A_{c}$ is a Schur algebra over $R$. This gives a map $\mathrm{S}\left(R_{c}\right) \rightarrow \mathrm{S}(R)$ and we let $R \otimes_{R_{c}} \mathrm{~S}\left(R_{c}\right)$ denote its image. Similarly, we have a map $\mathrm{S}\left(K_{c}\right) \rightarrow \mathrm{S}(K)$. Proposition 4.6. [22, p. 46] then asserts that $\mathrm{S}(K)=K \otimes_{K_{c}} \mathrm{~S}\left(K_{c}\right)$. This has been generalized by C. R. Riehm who proves that $\mathrm{S}(R)=R \otimes_{R_{c}} \mathrm{~S}\left(R_{c}\right)$, for any number ring $R$ (cf. [16, Theorem 1]). Thereby, the computation of $\mathrm{S}(R)$ is reduced to the subcyclotomic case, as it was for the Schur group over fields.

Lemma 1.4. Let $K \subseteq L$ be an extension of number fields, with rings of integers $R$ and $S$ respectively. Then an algebra $A$ is Azumaya over $R$ if and only if $S \otimes A$ is Azumaya over $S$.

Proof. If $A$ is Azumaya over $R$, then $S \otimes A$ is Azumaya over $S$, by a classical theorem on Azumaya algebras. Conversely, if $A$ is not Azumaya, then $\operatorname{disc}(A / R) \neq R, \operatorname{disc}(S \otimes A / S)=\operatorname{disc}(A / R) S \neq S$, and $S \otimes A$ is not Azumaya over $S$.

Theorem 1.5. The Schur group conjecture is true for number rings if and only if it is true for the number rings of quadratic fields $\mathbb{Q}(\sqrt{m}), m$ a squarefree natural number.

Proof. One direction is obvious, so let $R$ be any number ring and assume that the Schur group conjecture is true for $R=\mathbb{Z}[\rho]$, where $\rho=\sqrt{m}$ if $m \equiv 2$ or $3 \bmod 4$ and $\rho=(1+\sqrt{m}) / 2, m \equiv 1 \bmod 4, m$ squarefree.

Let $R$ be the number ring of a subcyclotomic extension $K$ and let [ $A$ ] be any element of $\mathrm{S}(K) \cap \operatorname{Br}(R)$. Then the Hasse invariants of [A] at the finite places are all 0 , since $[A] \in \operatorname{Br}(R)$. (Cf. [13, Corollary 6.35, p. 77].) On the other hand, the Hasse invariants at the infinite places are all equal, by the BenardSchacher theorem (cf. [22, Theorem 6.1, p. 89]). By Hasse's sum theorem, $\operatorname{Br}(R) \cap \mathrm{S}(K)=0$, unless $K$ has at least 2 and an even number of real places. In that case $\operatorname{Br}(R) \cap \mathrm{S}(K) \subseteq C_{2}$, the cyclic group of order 2. So we may further assume that $K$ has $2 p$ real embeddings, $p \in \mathbb{N} \backslash\{0\}$. Since $K$ is Galois over $\mathbb{Q}$ with abelian Galois group, this implies that $[K: \mathbb{Q}]$ is even and that there exist a quadratic extension $L$ of $\mathbb{Q}$ contained in $K$. Let $S$ denote the number ring of $L$. By our assumption, there exists a Schur algebra $A$ over $S$ such that $\operatorname{Inv}_{P}[K \otimes A]=1 / 2$ at any of the 2 infinite places $P$ of $L$. Then $R \otimes_{S} A$ is a Schur algebra over $R$ such that $\operatorname{Inv}_{Q}\left[K \otimes_{S} A\right]=1 / 2$ at any of the infinite places $Q$ of $K$. So $\mathrm{S}(R) \neq 0$ and the Schur group conjecture holds for $R$. Note that this also shows that $\operatorname{Br}(R) \cap S(K)=C_{2}$ if $K$ has an even number of real places.

\section{The SCHUR GROUP CONJECTURE FOR QUADRATIC NUMBER FIELDS}

Before providing a proof in the quadratic case, we give some additional definitions and recall some representation theory. As a general reference for the latter see [3] or [18]. Let $\eta$ be an irreducible character of a finite group such 
that $\eta(G) \subseteq K$. Then the center of the central simple component $B$ of $K G$ corresponding to $\eta$ is $K$. Let $\pi: K G \rightarrow B$ be the projection corresponding to $\eta$.

Definition 2.1. With the above notations, an irreducible character $\eta$ of $G$ is called an $R$ Azumaya character if and only if the $R$ order generated by $\pi(G)$ is Azumaya. The Hasse invariant of $\eta$ at any place is then equal to the Hasse invariant at that place of the Azumaya algebra corresponding to $\eta$.

To any Schur algebra $A$ over $R$ we can associate its Azumaya character $\eta$. Conversely, if $\eta$ is an Azumaya character, then for any representation $\pi: K G \rightarrow B$ as above, the $R$ order generated by $\pi(G)$ is Azumaya. Indeed, two such representations differ only by inner conjugation with an element of $B^{*}$, so the corresponding $R$ orders are actually isomorphic. Now let $P \in \operatorname{Spec}(R)$ be any prime ideal and assume that $K$ is sufficiently large with respect to $G$, i.e. $K$ contains the $\exp (G)$ th roots of unity. As already mentioned, $k$ denotes the quotient $R / P$. Then it is possible to define a decomposition map from the ring of $K$ characters of $G, \mathrm{Ch}(K)$, to the ring of Brauer characters, $\mathrm{Ch}(k)$ :

$$
d: \mathrm{Ch}(K) \rightarrow \mathrm{Ch}(k): \chi_{i} \mapsto \sum_{j=1}^{r} d_{i j} \phi_{j} .
$$

Here, the $\chi_{i}$ are irreducible $K$ characters and the $\phi_{j}$ are the Brauer characters of the simple $k(G)$ modules. The decomposition numbers $d_{i j}$ are then characterized by the relation $\chi_{i}(g)=\sum_{j=1}^{r} d_{i j} \phi_{j}(g)$ for any $P$ regular $g \in G$, i.e. for $g \in G$ such that $\operatorname{ord}(g) \notin P$. If $V$ is a $K G$ module with character $\eta$ and $N$ is any $G$ invariant $R$ lattice, then $d_{i j}$ is the multiplicity of the simple $k$ module with Brauer character $\phi_{j}$ occurring in a Jordan decomposition of the $k(G)$ module $k \otimes N$. In particular, if $\chi_{i}(g)=\phi_{j}(g)$ for all $P$ regular $g$, then the $k(G)$ module $k \otimes N$ is simple.

If $L$ is an extension of $K$ and $S$ is the integral closure of $R$ in $L$, then we let $l$ denote the quotient of $S$ by some prime ideal $P \in \operatorname{Spec}(S)$.

Theorem 2.2. Let $\eta$ be an irreducible character of $G$ such that $\eta(G) \subseteq K$. Let $L$ be any extension of $K$ such that $L$ is sufficiently large with respect to $G$ and let $S$ be the ring of integers of $L$. Then the following two assertions are equivalent:

(i) $\eta$ is an $R$ Azumaya character.

(ii) For any prime $P \in \operatorname{Spec}(S)$ and any $S G$ invariant lattice $N$ with character $\eta$, the module $l \otimes N$ is a simple $l(G)$ module.

Proof. (i) $\Rightarrow$ (ii) If $\eta$ is an $R$ Azumaya character, then it is also an $S$ Azumaya character, by Lemma 1.4. Let $\pi: L G \rightarrow B$ denote the projection on the central simple component corresponding to $\eta$ and let $\Gamma$ denote the $S$ order generated by $\pi(G)$. Then $\Gamma$ is an Azumaya algebra in $B$ and $N$ is a left $\Gamma$ module. For any prime $P \in \operatorname{Spec}(S)$, we have a commutative diagram

$$
\begin{array}{ccccc}
S G & \rightarrow & \Gamma & \rightarrow & \operatorname{End}_{S}(N) \\
\downarrow & & \downarrow & & \downarrow \\
l(G) & \rightarrow & l \otimes_{S} \Gamma & \rightarrow & \operatorname{End}_{l}\left(l \otimes_{S} N\right) .
\end{array}
$$

Since $\Gamma$ is Azumaya, $l \otimes_{S} \Gamma$ is central simple over the finite field $l$. Furthermore, since $L$ is sufficiently large, it has the same dimension as $\operatorname{End}_{l}(l \otimes N)$, 
so the map $l \otimes_{S} \Gamma \rightarrow \operatorname{End}_{l}\left(l \otimes_{S} N\right)$ is an isomorphism. This shows that $l \otimes_{S} N$ is a simple $l(G)$ module.

(ii) $\Rightarrow($ i) . Since $S$ is sufficiently large for $G$, any simple $l(G)$ module is absolutely simple, i.e. it remains simple under any field extension. By a theorem of Burnside (cf. [3, Theorem 3.43, p. 54]) this implies that the map $l \otimes_{S} \Gamma \rightarrow \operatorname{End}_{l}\left(l \otimes_{S} N\right)$ in the above diagram is an epimorphism. A comparison of the dimensions over $l$ then shows that this map is injective too, so $l \otimes_{S} \Gamma$ is a central simple $l$ algebra, for any $P \in \operatorname{Spec}(S)$. By Theorem 7.1 of [4] this implies that $\eta$ is an $S$ Azumaya character and by applying Lemma 1.4 that it is an $R$ Azumaya character too.

Let $L$ be a cyclotomic extension of the number field $K$, with Galois group $\mathscr{G}$ and let $c$ be a 2-cocycle with values in $t\left(L^{*}\right)$, where $t\left(L^{*}\right)$ is the group of roots of unity in $L^{*}$. If $\mathbb{S}$ is any cofinite set of places of $K$, then we let $R_{\mathbb{S}}$ denote the ring of $\mathbb{S}$ integers of $K$ and $S_{\mathbb{S}}$ the integral closure of $R_{\mathbb{S}}$ in $L$. We can use $c$ to construct a crossed product order $\left(S_{\mathbb{S}}, \mathscr{G}, c\right)=\bigoplus_{\sigma \in \mathscr{G}} R u_{\sigma}$ by the rules

$$
u_{\sigma} \cdot u_{\tau}=c(\sigma, \tau) u_{\sigma \tau}, \quad u_{\sigma} \cdot s=\sigma(s) \cdot u_{\sigma} .
$$

The following theorem is an adapted version of a theorem of Auslander, Goldman, and Rim.

Theorem 2.3. The crossed product algebra $\left(S_{\mathbb{S}}, \mathscr{G}, c\right)$ is an $R_{\mathbb{S}}$ Schur algebra if and only if the extension $L / K$ is unramified at all finite places in $\mathbb{S}$.

Proof. The extension $S_{\mathbb{S}} / R_{\mathbb{S}}$ is a Galois extension if and only if the condition on $L / K$ is satisfied. By a straightforward adaptation of Theorem 40.14 , [14, p. 374], $\left(S_{\mathbb{S}}, \mathscr{G}, c\right)$ is an Azumaya algebra over $R_{\mathbb{S}}$ if and only if $S_{\mathbb{S}}$ is a Galois extension of $R_{\mathbb{S}}$. We still have to verify that $\left(S_{\mathbb{S}}, \mathscr{G}, c\right)$ is a Schur algebra. If $L=K\left[\varepsilon_{n}\right]$ then it is an easy exercise to construct an epimorphism $S_{\mathbb{S}}\left(t\left(L^{*}\right) \rtimes_{c} \mathscr{G}\right)$ onto $\left(S_{\mathbb{S}}, \mathscr{G}, c\right)$.

Example 2.4. Let $p$ be any odd prime, $\kappa=(-1)^{(p-1) / 2}$ and let $K=\mathbb{Q}(\sqrt{\kappa p})$, $L=K\left(\varepsilon_{p}\right)$. It is well known that $L / K$ is only ramified at the prime $(\sqrt{p})$ of $K$. Let $R$ denote the number ring of $K$ and let $R_{Q}$ denote its localization at any prime $Q \in \operatorname{Spec}(R)$. Applying Theorem 2.3, we see that the crossed product order $R_{Q} \otimes\left(\mathbb{Z}\left[\varepsilon_{p}\right], \mathscr{G}, c\right)$ is Azumaya for any prime $Q$ of $\operatorname{Spec}(R)$ not containing $p$.

Let $m$ be any squarefree number. We say that $\mathscr{S}(m)$ is true if and only if the Schur group conjecture is true for the number field $K=\mathbb{Q}(\sqrt{m})$ and its number ring $R$. We let $\lambda_{m}$ denote the nontrivial element of $\operatorname{Gal}(K / \mathbb{Q})$, where we also allow $m$ to be negative.

Example 2.5. $\mathscr{S}(2)$ is true. Let $A$ be the $\mathbb{Z}[\sqrt{2}]$ order of the quaternion algebra $(-1,-1)$ over $\mathbb{Q}(\sqrt{2})$ generated by $<1,(1+i) / \sqrt{2},(1+j) / \sqrt{2}$, $(1+i+j+k) / 2>$ as a $\mathbb{Z}[\sqrt{2}]$ module. In [21] it is proved that $A$ is the epimorphic image of $E_{48}$, the binary tetraedral group of order 48 . More generally, the finite subgroups of the invertible elements of skew fields have been determined by Amitsur in [1]. Let $E_{24}=\{ \pm 1, \pm i, \pm j, \pm k,( \pm 1+ \pm i+ \pm j+ \pm k) / 2\}$ then $E_{48}=E_{24} \cup\{x / \sqrt{2}, x= \pm y \pm z$ and $y, z \in\{1, i, j, k\}, y \neq z\}$. Moreover, $E_{24}$ is a normal subgroup of $E_{48}$. An easy calculation shows that $\operatorname{disc}(A / \mathbb{Z}[\sqrt{2}])=1$, so $A$ is Azumaya and $\mathscr{S}(2)$ is true. If $\eta_{2}$ denotes the 
irreducible character corresponding to this representation and tr denotes the reduced trace on $A$, then by definition of the reduced trace, $\eta_{2}(g)=\operatorname{tr}(g)$ for any $g \in E_{48}$. Let $\xi_{2}$ be the automorphism defined on $E_{48}$ by $\xi_{2} \mid E_{24}=$ id, $\xi_{2}(x / \sqrt{2})=-x / \sqrt{2}$, then $\lambda_{2}\left(\eta_{2}(g)\right)=\eta_{2}\left(\xi_{2}(g)\right)$ for any $g \in E_{48}$.

Now let $p$ be any odd prime and let $\mathbb{F}_{p}$ denote the finite Galois field of order $p$. In order to prove that $\mathscr{S}(p)$ is true, we need some facts concerning $\mathrm{Sl}_{2}\left(\mathbb{F}_{p}\right)$ and its representations. For a character table of $\mathrm{Sl}_{2}\left(\mathbb{F}_{p}\right)$ we refer to [7, p. 228]. Let $\kappa=(-1)^{(p-1) / 2}, \alpha$ any nonsquare in $\mathbb{F}_{p}^{*}, \nu$ any generator of $\mathbb{F}_{p}^{*}$, and $\tau$ any generator of $\mathbb{F}_{p^{2}}$. Fix a basis of $\mathbb{F}_{p^{2}}$ as an $\mathbb{F}_{p}$ vector space. Once these choices have been made, we determine elements $z, c, d, a$ and $b$ by

$$
z=\left(\begin{array}{cc}
-1 & 0 \\
0 & -1
\end{array}\right) \quad c=\left(\begin{array}{ll}
1 & 1 \\
0 & 1
\end{array}\right) \quad d=\left(\begin{array}{ll}
1 & \alpha \\
0 & 1
\end{array}\right) \quad a=\left(\begin{array}{cc}
\nu & 0 \\
0 & \nu^{-1}
\end{array}\right)
$$

and $b$ is the matrix of $\mathbb{F}_{p^{2}} \rightarrow \mathbb{F}_{p^{2}}: x \mapsto \tau^{p-1} x$ with respect to the chosen basis. A complete set of representatives of the conjugacy classes of $\mathrm{Sl}_{2}\left(\mathbb{F}_{p}\right)$ is then given by

$$
\begin{aligned}
(1),(z),(c),(d),(z c),(z d),\left(a^{l}\right),\left(b^{m}\right) \\
\text { for } 1 \leq l \leq(p-3) / 2 ; 1 \leq m \leq(p-1) / 2
\end{aligned}
$$

We will need only a small portion of the character table of $\mathrm{Sl}_{2}\left(\mathbb{F}_{p}\right)$.

\begin{tabular}{|c|c|c|c|c|c|c|c|c|}
\hline & 1 & $z$ & $c$ & $d$ & $z c$ & $z d$ & $a^{l}$ & $b^{m}$ \\
\hline$\eta_{p}$ & $\frac{p-1}{2}$ & $\frac{-\kappa(p-1)}{2}$ & $\frac{-1+\sqrt{\kappa p}}{2}$ & $\frac{-1-\sqrt{\kappa p}}{2}$ & $\frac{\kappa(1-\sqrt{\kappa p})}{2}$ & $\frac{\kappa(1+\sqrt{\kappa p})}{2}$ & 0 & $(-1)^{m+1}$ \\
$\eta_{p}^{\prime}$ & $\frac{p-1}{2}$ & $\frac{-\kappa(p-1)}{2}$ & $\frac{-1-\sqrt{\kappa p}}{2}$ & $\frac{-1+\sqrt{\kappa p}}{2}$ & $\frac{\kappa(1+\sqrt{\kappa p})}{2}$ & $\frac{\kappa(1-\sqrt{\kappa p})}{2}$ & 0 & $(-1)^{m+1}$ \\
\hline
\end{tabular}

Let $\xi_{p}$ be the outer automorphism on $\mathrm{Sl}_{2}\left(\mathbb{F}_{p}\right)$ defined by inner conjugation with $\left(\begin{array}{ll}\nu & 0 \\ 0 & 1\end{array}\right)$ then from the above table we find

$$
\forall g \in \mathrm{Sl}_{2}\left(\mathbb{F}_{p}\right): \lambda_{\kappa p}\left(\eta_{p}(g)\right)=\eta_{p}\left(\xi_{p}(g)=\eta_{p}^{\prime}(g) .\right.
$$

The absolutely simple $\mathrm{Sl}_{2}\left(\mathbb{F}_{p}\right)$ modules in characteristic $p$ may be described as follows (cf. [3, Example 17.17, p. 426]). Let $V_{i}$ be the vector space of homogeneous polynomials of degree $i$ in $X, Y$ over $\mathbb{F}_{p}, 0 \leq i \leq p-1$. The action of $\mathrm{Sl}_{2}\left(\mathbb{F}_{p}\right)$ on $V_{i}$ is given by

$$
\left(\begin{array}{ll}
\alpha & \beta \\
\gamma & \delta
\end{array}\right)\left(X^{l} Y^{m}\right)=(\alpha X+\beta Y)^{l}(\gamma X+\delta Y)^{m}
$$

These are all simple $\mathrm{Sl}_{2}\left(\mathbb{F}_{p}\right)$ modules, up to isomorphism. We are now ready to prove

Theorem 2.6. $\eta_{p}$ and $\eta_{p}^{\prime}$ are $\mathbb{Z}[(1+\sqrt{\kappa p}) / 2]$ Azumaya characters.

Proof. The proofs for $\eta_{p}$ and $\eta_{p}^{\prime}$ are entirely similar, so let $\eta$ stand for any of them. Let $R=\mathbb{Z}[(1+\sqrt{\kappa p}) / 2], K=\mathbb{Q}(\sqrt{\kappa p})$ and let $\pi: K \mathrm{Sl}_{2}\left(\mathbb{F}_{p}\right) \rightarrow B$ be the projection on the $K$ central simple component corresponding to $\eta$. The $R$ order generated by $\eta\left(\mathrm{Sl}_{2}\left(\mathbb{F}_{p}\right)\right)$ will be denoted $\Gamma$. In order to prove that $\Gamma$ is Azumaya, it suffices to prove this at any localization of $R$ (cf. [4, Theorem 7.1, p. 72]). Let $H$ be the subgroup of $\mathrm{Sl}_{2}\left(\mathbb{F}_{p}\right)$ consisting of the upper triangular matrices, $\Delta$ the $R$ order generated by $\eta(H), u_{\sigma}=\eta(a)$, and $\varepsilon_{p}=\eta(c)$. Then $\varepsilon_{p}$ is indeed a $p$ th root of unity. Since $u_{\sigma} \varepsilon_{p}=\varepsilon_{p}^{\nu^{2}} u_{\sigma}$ 
and $\nu$ is a generator of $\mathbb{F}_{p}^{*}$, this shows that inner conjugation by $u_{\sigma}$ generates $\operatorname{Gal}\left(\mathbb{Q}\left(\varepsilon_{p}\right) / \mathbb{Q}(\sqrt{\kappa p})\right)$. Therefore, $\Delta \cong\left(R\left[\varepsilon_{p}\right], \operatorname{Gal}\left(K\left(\varepsilon_{p}\right) / K, c\right)\right.$ for some 2cocycle $c$. Example 2.4 then shows that $R_{Q} \otimes \Delta$ is Azumaya for any prime $Q \in \operatorname{Spec}(R)$ such that $p \notin Q$. Then $R_{Q} \otimes \Gamma=R_{Q} \otimes \Delta$, since Azumaya algebras are maximal orders (cf. [13, Theorem 6.18, p. 66]), which shows that $R_{Q} \otimes \Gamma$ is also Azumaya. Let $P$ be the unique prime of $R$ lying above $p$ and let $S$ be an extension of $R$ that is sufficiently large for $\mathrm{Sl}_{2}\left(\mathbb{F}_{p}\right)$. By Lemma 1.4 , it suffices to prove that $S_{p} \otimes \Gamma$ is Azumaya. If $P^{\prime}$ is any prime of $S$ lying above $P$, then after localizing further we have to prove that $S_{P^{\prime}} \otimes \Delta$ is Azumaya. By Theorem 2.2 this is equivalent to the assertion that $\eta$ has the same values on the $P^{\prime}$ regular elements of $g \in \mathrm{Sl}_{2}\left(\mathbb{F}_{p}\right)$ as the Brauer character of some absolutely simple $\mathrm{Sl}_{2}\left(\mathbb{F}_{p}\right)$ module in characteristic $p$. Let $\varphi_{i}$ denote the Brauer character of the simple module $V_{i}$. By comparing the values in 1 , we see that we have to verify $\eta(g)=\varphi_{(p-3) / 2}(g)$ for any $P^{\prime}$ regular $g \in \mathrm{Sl}_{2}\left(\mathbb{F}_{p}\right)$, that is for $g \in\left\{1, z, a^{l}, b^{m}\right\}$. The verification for $1, z$ is trivial and that for $a^{l}$ will be left to the diligent reader. We will verify this for $b^{m}$. Define $\tilde{b} \in \mathrm{Sl}_{2}\left(\mathbb{F}_{p^{2}}\right)$ by

$$
\tilde{b}: \mathbb{F}_{p^{2}} \otimes V_{(p-3) / 2} \rightarrow \mathbb{F}_{p^{2}} \otimes V_{(p-3) / 2}: X \mapsto \tau^{(p-1)} X, Y \mapsto \tau^{(1-p)} Y .
$$

Then $b^{m}$ and $\tilde{b}^{m}$ have the same characteristic equation which is separable over $\mathbb{F}_{p^{2}}$ and therefore they are conjugate in $\mathrm{Gl}_{2}\left(\mathbb{F}_{p^{2}}\right)$. So we may replace $b^{m}$ by $\tilde{b}^{m}$ in the calculation of $\varphi_{(p-3) / 2}\left(b^{m}\right)$. Let $\omega$ be a $(p+1)$ th root of unity.

$$
\begin{aligned}
\varphi_{(p-3) / 2}\left(b^{m}\right) & =\sum_{i=0}^{i=(p-3) / 2} \omega^{m i} \omega^{-m((p-3) / 2-i)} \\
& =\omega^{-m((p-3) / 2)} \frac{\omega^{m(p-1)}-1}{\omega^{2 m}-1}=(-1)^{m} \omega^{2 m} \frac{\omega^{m(p-1)}-1}{\omega^{2 m}-1}=\eta\left(b^{m}\right) .
\end{aligned}
$$

This finishes the proof that $\eta$ is Azumaya.

Corollary 2.7. $\mathscr{S}(p)$ is true if $p$ is a prime such that $p \equiv 1 \bmod 4$.

Proof. Let us resume the notations of the above theorem, then we already know that $\eta_{p}$ is an $R$ Azumaya algebra. We have to calculate the Hasse indices at the two infinite places of $K$. But $K \otimes \Gamma=K \otimes \Delta$ and $K \otimes \Delta \cong\left(K\left(\varepsilon_{p}\right)\right.$, $\operatorname{Gal}\left(K\left(\varepsilon_{p}\right) / K=\langle\sigma\rangle, a\right)$, a cyclic algebra over $K$. Here $a=u_{\sigma}^{(p-1) / 2}=-1$, since $\eta_{p}$ is a faithful $\mathrm{Sl}_{2}\left(\mathbb{F}_{p}\right)$ character. If $K \hookrightarrow \mathbb{R}$ is any real place of $K$, then $\mathbb{R} \otimes_{K}(K \otimes \Delta)$ and $\left(\mathbb{R}, \sigma^{(p-1) / 4},-1\right)$ are Brauer equivalent (cf. [14, Theorem 30.8$, p. 261] $)$ and $\operatorname{Inv}\left(\mathbb{R}, \sigma^{(p-1) / 4},-1\right)=1 / 2$.

Corollary 2.8. The Schur indices of $\eta_{p}$ and $\eta_{p}^{\prime}$ are 0 at all finite places.

Proof. $\operatorname{Br}(R)$ consists of those classes of central simple algebras for which the invariant vanishes at the finite places of $R$.

Corollary 2.8 has already been proved by G. J. Janusz in [10] and by W. Feit in [8].

Remark 2.9. An explicit construction of the characters of $\mathrm{Sl}_{2}\left(\mathbb{F}_{p}\right)$ over $\mathbb{C}$ has been given by $S$. Tanaka in [19]. It makes use of a projective representation of the Heisenberg group of $\mathbb{F}_{p^{2}}$, which was discovered by A. Weil. 
If $p \equiv 3 \bmod 4$, then Theorem 2.6 cannot be applied immediately to construct nontrivial elements of $S(\mathbb{Z}[\sqrt{p}])$. However, we will give a construction based on Theorem 2.6 to prove that $\mathscr{S}(p)$ is also true in this case. Since this construction is a particular case of a more general one, we postpone this proof for the moment.

Definition 2.10. A brick is a sextupel $(G, m, \eta, g, \xi, x)$ consisting of the following data:

(i) A finite group $G$.

(ii) A squarefree $m \in \mathbb{Z}$.

(iii) An irreducible Azumaya character $\eta$ on $G$ with values in $\mathbb{Q}(\sqrt{m})$.

(iv) A element $g \in G$ satisfying $\operatorname{ord}(g)=|m|^{\alpha}$ for some $\alpha \in \mathbb{N} \backslash\{0\}$, $\eta(g) \notin \mathbb{Q}$

(v) An automorphism $\xi$ on $G$ such that $\forall g \in G: \lambda_{m}(\eta(g)=\eta(\xi(g))$

(vi) An element $x$ in $G$ such that $\xi^{2}$ is inner conjugation by $x$ and $x$ is invariant under $\xi$. If, in condition (iv), we suppress the requirement that $\operatorname{ord}(g)=|m|^{\alpha}$, then we call the sextupel a semibrick.

As the name suggests, these bricks allow us to construct new Azumaya characters from old ones. If $\xi$ is an automorphism of $G$ as in the above definition, then we can extend $G$ to a new group, which we denote by $E(G, \xi)$ as follows. As a set, $E(G, \xi)=G \times\{1, \xi\}$ and the multiplication is determined by the following rules

$$
\begin{gathered}
(g, 1)\left(g^{\prime}, 1\right)=\left(g g^{\prime}, 1\right), \quad(g, 1)(1, \xi)=(g, \xi), \\
(1, \xi)(g, 1)=(\xi(g), \xi), \quad(1, \xi)^{2}=(x, 1) .
\end{gathered}
$$

If $\eta$ is a character on $G$, then its induction to $E(G, \xi)$ will be denoted by $E(\eta)$. Note that $E(\eta) \mid G=\eta+\eta \circ \xi$ by Mackey's subgroup theorem (cf. [3, 10.14, p. 238]) and $E(\eta)$ vanishes outside $G$.

Example 2.11. $\mathscr{B}(2)=\left(E_{48}, 2, \eta_{2},(1+i) / \sqrt{2}, \xi_{2}, 1\right)$ is a brick. This is a consequence of Example 2.5 and the discussion following it. For any odd prime $p, \mathscr{B}(p)=\left(\mathrm{Sl}_{2}\left(\mathbb{F}_{p}\right), \kappa p, \eta_{p}, c, \xi_{p}, a\right)$ is a brick. This follows from Theorem 2.6 and the properties of $\mathrm{Sl}_{2}\left(\mathbb{F}_{p}\right)$ mentioned above. Similarly, if $\gamma$ denotes the nontrivial character of $C_{2}$, then $\mathscr{B}^{\prime}(p)=\left(\{ \pm 1\} \times \mathrm{Sl}_{2}\left(\mathbb{F}_{p}\right), \kappa p, \gamma \otimes \eta_{p},(1, c)\right.$, id $\left.\times \xi_{p},(-1, a)\right)$ is a brick. Finally, let $E_{48}^{\prime}$ be the group generated by:

$$
\left\{\frac{(1+i)}{\sqrt{-2}}, j\right\} \subseteq\left(\begin{array}{c}
i j \\
\mathbb{Q}(\sqrt{-2})
\end{array}\right)^{*}
$$

Let $\eta_{-2}$ be the corresponding character and set $\xi_{-2} \mid E_{24}=$ id and $\xi_{-2}(x / \sqrt{-2})$ $=-x / \sqrt{-2}$. Then $\mathscr{B}^{\prime}(2)=\left(E_{48}^{\prime},-2, \eta_{-2},(1+i) / \sqrt{-2}, \xi_{-2}, 1\right)$ is a brick. We use these bricks later on.

\section{Theorem 2.12.}

(i) If $(G, m, \eta, g, \xi, x)$ is a brick, then so is $(G \times G, m, \eta \otimes \eta,(g, 1), \xi \times$ $\xi,(x, x))$.

(ii) If $\left(G_{1}, m_{1}, \eta_{1}, g_{1}, \xi_{1}, x_{1}\right)$ and $\left(G_{2}, m_{2}, \eta_{2}, g_{2}, \xi_{2}, x_{2}\right)$ are bricks, and g.c.d. $\left(m_{1}, m_{2}\right)=1$ then so is $\left(E\left(G_{1} \times G_{2}, \xi_{1} \times \xi_{2}\right), m_{1} m_{2}\right.$, $\left.E\left(\eta_{1} \otimes \eta_{2}\right),\left(g_{1}, g_{2}\right), E\left(\xi_{1} \times 1\right),\left(x_{1}, 1\right)\right)$. 
Proof. (i) Let $R, K, \Gamma$ and $\pi$ have the same meaning as in the proof of Theorem 2.6, with $\eta_{p}$ replaced by $\eta$. Then $\pi \otimes \pi: K G \otimes K G \cong K(G \times G) \rightarrow B \times B$ corresponds to $\eta \otimes \eta$ and the $R$ order spanned by $(\pi \otimes \pi)(G \times G)$ is isomorphic to $\Gamma \otimes \Gamma$. Since the tensor product of two Azumaya algebras is Azumaya, this shows that $\eta \otimes \eta$ is an $R$ Azumaya character. The other constituents of the brick are easily seen to verify the required properties.

(ii) Since $x_{1}$ is left invariant under $\xi_{1}$, we can extend $\left(\xi_{1} \times 1\right)$ to $E\left(G_{1} \times G_{2}, \xi_{1} \times \xi_{2}\right)$ by

$$
E\left(\xi_{1} \times 1\right):\left(g_{1}, g_{2},\left(\xi_{1} \times \xi_{2}\right)^{\mu}\right) \mapsto\left(\xi_{1}\left(g_{1}\right), g_{2},\left(\xi_{1} \times \xi_{2}\right)^{\mu}\right) \quad \mu=0,1 .
$$

This explains the appearance of $E\left(\xi_{1} \times 1\right)$ in the brick.

Clearly, $E\left(\eta_{1} \otimes \eta_{2}\right)$ takes values in $\mathbb{Q}\left(\sqrt{m_{1}}, \sqrt{m_{2}}\right)$. Let $\lambda_{m_{i}}$ also denote the extension to $\mathbb{Q}\left(\sqrt{m_{1}}, \sqrt{m_{2}}\right)$ which fixes $\sqrt{m_{3-i}}$ and let $\tau=\lambda_{m_{1}} \circ \lambda_{m_{2}}$. Then

$$
\begin{aligned}
\tau \circ E\left(\eta_{1} \otimes \eta_{2}\right)\left(g_{1}, g_{2}, 1\right) & =\tau\left(\eta_{1}\left(g_{1}\right) \eta_{2}\left(g_{2}\right)+\eta_{1}\left(\xi_{1}\left(g_{1}\right)\right) \eta_{2}\left(\xi_{2}\left(g_{2}\right)\right)\right) \\
& =\tau\left(\eta_{1}\left(g_{1}\right) \eta_{2}\left(g_{2}\right)+\tau \eta_{1}\left(g_{1}\right) \tau \eta_{2}\left(g_{2}\right)\right) \\
& =E\left(\eta_{1} \otimes \eta_{2}\right)\left(g_{1}, g_{2}, 1\right) .
\end{aligned}
$$

This shows that $E\left(\eta_{1} \otimes \eta_{2}\right)$ takes values in $\mathbb{Q}\left(\sqrt{m_{1} m_{2}}\right)$, as required. By an entirely similar calculation, one proves that

$$
\lambda_{m_{1} m_{2}} \circ E\left(\eta_{1} \otimes \eta_{2}\right)\left(g_{1}, g_{2}, 1\right)=E\left(\eta_{1} \otimes \eta_{2}\right)\left(\xi_{1}\left(g_{1}\right), g_{2}, 1\right)
$$

and

$$
E\left(\eta_{1} \otimes \eta_{2}\right)\left(g_{1}, g_{2}, 1\right) \notin \mathbb{Q} .
$$

All the other properties needed to form a brick are now rather obvious, except that we have to prove that $E\left(\eta_{1} \otimes \eta_{2}\right)$ is an Azumaya character over the number ring of $\mathbb{Q}\left(\sqrt{m_{1} m_{2}}\right)$. To this end, we will use the criterion of Theorem 2.2 and replace $R$ by a number ring $S$ for which the quotient field is large enough for $G$. As in the first part of the proof, $\eta_{1} \otimes \eta_{2}$ is an $S$ Azumaya character. Let $N_{i}$ be an invariant $S G_{i}$ module with character $\eta_{i}, i=1,2$. The automorphism $\xi_{i}$ on $G_{i}$ may be used to change the module structure on $N_{i}$. The resulting module will be denoted by $N_{i}^{\xi_{i}}$. Let $L_{1}=N_{1} \otimes N_{2}$ and $L_{2}=N_{1}^{\xi_{1}} \otimes N_{2}^{\xi_{2}}=\left(N_{1} \otimes N_{2}\right)^{\xi_{1} \otimes \xi_{2}}$, then

$$
E\left(N_{1} \otimes N_{2}\right)=\operatorname{Ind}_{G_{1} \times G_{2}}^{E\left(G_{1} \times G_{2}, \xi_{1} \times \xi_{2}\right)}\left(L_{1}\right), E\left(N_{1} \otimes N_{2}\right) \mid G_{1} \times G_{2}=L_{1} \oplus L_{2}
$$

by one of the many forms of Mackey's subgroup theorem (cf. [3, 10.14, p. 238]).

Now let $P$ be any prime of $S$ and assume that $m_{2} \notin P$. Since $\eta_{1} \otimes \eta_{2}$ is an Azumaya character, Theorem 2.2 shows that $l \otimes L_{1}$ is a simple $G_{1} \otimes G_{2}$ module with Brauer character $\eta_{1} \otimes \eta_{2} \mid\left(G_{1} \times G_{2}\right)_{\text {reg }}$. Now $m_{2} \notin P$ implies that $\left(1, g_{2}, 1\right) \in E\left(G_{1} \times G_{2}\right)$ is a $P$ regular element and

$$
\left(\eta_{1} \otimes \eta_{2}\right)\left(1, g_{2}, 1\right) \neq\left(\eta_{1} \otimes \eta_{2}\right)\left(1, \xi_{2}\left(g_{2}\right), 1\right) .
$$

This shows that $l \otimes L_{1}$ and $l \otimes L_{2}$ have different Brauer characters and therefore, are nonisomorphic simple $l\left(G_{1} \times G_{2}\right)$ modules. If $M$ is any $E\left(G_{1} \times G_{2}, \xi_{1} \times \xi_{2}\right)$ invariant submodule of $E\left(N_{1} \otimes N_{2}\right)$, then $M$ is also $l\left(G_{1} \times G_{2}\right)$ invariant, and we have $M=\oplus_{j \in \mathcal{J}} L_{j}$, for some subset $\mathcal{J}$ of $\{1,2\}$ (cf. [3, Proposition $3.20 \mathrm{iv}$, p. 46]). However, if $L_{j} \subseteq M$, then $\left(\xi_{1} \times \xi_{2}\right) L_{j}=L_{3-j} \subseteq M$. So $\mathscr{J}=\{1,2\}$ and $M=E\left(N_{1} \otimes N_{2}\right)$ which shows that $E\left(N_{1} \otimes N_{2}\right)$ is simple. 
The argument is clearly similar if $m_{1} \notin P$ and either $m_{1}$ or $m_{2}$ do not belong to $P$ by our assumption that g.c.d. $\left(m_{1}, m_{2}\right)=1$. Finally, the very same method applies to show that $E\left(\eta_{1} \otimes \eta_{2}\right)$ is irreducible.

Note that the above theorem is still valid if one replaces bricks by semibricks. However, one has to require in this case that g.c. d. $\left(\operatorname{ord}\left(g_{1}\right), \operatorname{ord}\left(g_{2}\right)\right)=1$.

Corollary 2.13. If $p$ is an odd prime, $p \equiv 3 \bmod 4$, then $\mathscr{S}(p)$ is true.

Proof. Let $C_{4}=\langle x\rangle$ be the cyclic group or order $4, \eta_{-1}$ a linear character such that $\eta_{-1}(x)=i$ and $\xi_{-1}$ the unique nontrivial automorphism on $C_{4}$. Since $\eta_{-1}$ is a linear character, it is clear that $\eta_{-1}$ is a $\mathbb{Z}[i]$ Azumaya character. Therefore $\left(C_{4},-1, \eta_{-1}, x, \xi_{-1}, x^{2}\right)$ is a semibrick. By our remark following Theorem $2.12, \mathscr{B}^{\prime \prime}(p)=\left(E\left(C_{4} \times \mathrm{Sl}_{2}\left(\mathbb{F}_{p}\right)\right), p, E\left(\eta_{-1} \times \eta_{p}\right),(x, c), \xi_{-1} \times 1\right.$, $\left.\left(x^{2}, 1\right)\right)$ is a semibrick too. This already provides us with a $\mathbb{Z}[\sqrt{p}]$ Azumaya character. We still have to calculate the index at any of the two infinite places of $\mathbb{Q}(\sqrt{p})$.

Let $\pi: \mathbb{Q}[\sqrt{p}] E\left(C_{4} \times \mathrm{Sl}_{2}\left(\mathbb{F}_{p}\right)\right) \rightarrow B$ denote the projection corresponding to $E\left(\eta_{-1} \times \eta_{p}\right)$ and let $\Delta$ be the $\mathbb{Z}[\sqrt{p}]$ order generated by $\pi\left(C_{4} \times \operatorname{Sl}_{2}\left(\mathbb{F}_{p}\right)\right)$. Let $\varepsilon_{4 p}=\pi(x, c, 1)=i \varepsilon_{p}$ and $u_{\sigma}=\pi\left(1,1, \xi_{-1} \times \xi_{p}\right)$. Then $u_{\sigma} \varepsilon_{4 p}=i^{3} \varepsilon_{p}^{\nu} u_{\sigma}$. But

$$
\sigma: \mathbb{Q}\left(\varepsilon_{4 p}\right) \rightarrow \mathbb{Q}\left(\varepsilon_{4 p}\right): i \mapsto i^{3} \quad \varepsilon_{p} \mapsto \varepsilon_{p}^{\nu}
$$

is a generator of $\operatorname{Gal}\left(\mathbb{Q}\left(\varepsilon_{4 p}\right) / \mathbb{Q}(\sqrt{p})\right)$. So

$$
\mathbb{Q}(\sqrt{p}) \otimes \Delta \cong\left(\mathbb{Q}\left(\varepsilon_{4 p}\right), \operatorname{Gal}\left(\mathbb{Q}\left(\varepsilon_{4 p}\right) / \mathbb{Q}(\sqrt{p})\right), a\right),
$$

where $a=u_{\sigma}^{p-1}$. However, $B$ and $\mathbb{Q}(\sqrt{p}) \otimes \Delta$ have the same dimension over $\mathbb{Q}(\sqrt{p})$, so they are equal. The assumption that $p \equiv 3 \bmod 4$ and the fact that $\xi_{-1}^{2}=x^{2}$ will after a little calculation show that $a=-1$. As in the proof of Corollary 2.7, it follows that the Hasse invariant at any of the infinite places is $1 / 2$.

To keep further track of the index at the infinite places, we will apply the following strong result due to Fontaine (cf. [9]).

Theorem 2.14. Let $A$ be a subgroup of the finite group $G$. Suppose $\varphi$ and $\gamma$ are irreducible characters of $A$ and $G$ respectively such that $(\varphi, \gamma)_{A}=1$. Let $K$ be a field such that $K=K(\gamma)=K(\varphi)$. Then the $K$ central simple components corresponding to $\varphi$ and to $\gamma$ are Brauer equivalent.

Lemma 2.15. Let $\mathscr{C}_{1}$ and $\mathscr{C}_{2}$ be two bricks or semibricks satisfying the conditions of Theorem 2.12 and assume further that the character constituents $\eta_{1}$ and $\eta_{2}$ of both $\mathscr{C}_{1} \times \mathscr{C}_{2}$ have real values. Let $E\left(\mathscr{C}_{1} \times \mathscr{C}_{2}\right)$ denote the brick or semibrick constructed in Theorem 2.12(ii). Then the Hasse invariant of $E\left(\eta_{1} \otimes \eta_{2}\right)$ is equal to the sum of the Hasse invariants of the $\eta_{i}, i=1,2$.

Proof. We apply Theorem 2.14 with the groups $G_{1} \times G_{2}$ and $E\left(G_{1} \times G_{2}\right)$, the characters $\eta_{1} \otimes \eta_{2}$ and $E\left(\eta_{1} \otimes \eta_{2}\right)$ and the field $\mathbb{R}$. Note that the conditions of the theorem are satisfied since

$$
\left(E\left(\eta_{1} \otimes \eta_{2}\right), \eta_{1} \otimes \eta_{2}\right)_{G_{1} \times G_{2}}=\left(\eta_{1} \otimes \eta_{2}+\eta_{1}^{\xi_{1}} \otimes \eta_{2}^{\xi_{2}}, \eta_{1} \otimes \eta_{2}\right)=1 .
$$

Here, we used the fact that $\eta_{1} \otimes \eta_{2}$ is irreducible and that $\xi_{1} \times \xi_{2}$ acts nontrivially on this character. Since the Hasse invariants of $\eta_{1} \otimes \eta_{2}$ are just the sum of the Hasse invariants of the $\eta_{i}$, we have proved that the Hasse invariants at infinity 
of $E\left(\eta_{1} \otimes \eta_{2}\right)$ are the sum of the Hasse invariants of the Azumaya algebras determined by $\mathscr{C}_{i}$.

Theorem 2.16. For any squarefree natural number $m, \mathscr{S}(m)$ is true.

Proof. Let $m=2^{\varepsilon} p_{1} \ldots p_{r} q_{1} \ldots q_{s}$, where $\varepsilon \in\{0,1\}, p_{i} \equiv 1 \bmod 4, q_{j} \equiv$ $3 \bmod 4$. For any prime $p$, let $\mathscr{B}(p), \mathscr{B}^{\prime}(p)$, respectively $\mathscr{B}^{\prime \prime}(p)$, have the same meaning as in Example 2.11, respectively Corollary 2.13. Note that the irreducible characters of the (semi) bricks $\mathscr{B}(2), \mathscr{B}\left(p_{i}\right), \mathscr{B}^{\prime \prime}\left(q_{j}\right)$ take values in $\mathbb{R}$ and that the Hasse invariants at the infinite places of the constructed Azumaya algebras are all $1 / 2$. If $\mathscr{C}_{1}$ and $\mathscr{C}_{2}$ are two bricks which satisfy the conditions of Theorem 2.12, then we let $\mathscr{C}_{1}^{2}$ denote the brick constructed in Theorem 2.12(i), starting from $\mathscr{C}_{1}$. Similarly, $\mathscr{C}_{1} \times \mathscr{C}_{2}$ denotes the brick obtained by applying the construction in Theorem 2.12(ii).

It is not difficult to prove, along the lines of Corollary 2.13, that the Azumaya algebras corresponding to $\mathscr{B}^{\prime}\left(q_{i}\right) \times \mathscr{B}\left(q_{j}\right)$ and to $\mathscr{B}^{\prime}(2) \times \mathscr{B}\left(q_{i}\right)$ have Hasse invariants $1 / 2$ at all infinite places. As we already know that $\mathscr{S}(t)$ is true for any prime $t$, we may assume that $r \neq 0$ or $s \neq 0$. We now construct semibricks $\mathscr{D}$ which depend on the values of the parameters $\varepsilon, r$, and $s$. For the construction of $\mathscr{D}$, the bricks are constructed from left to right, but squaring and bracketing take precedence.

$$
\begin{aligned}
& \varepsilon=0, s \text { odd: } \\
& \mathscr{D}=\mathscr{B}^{\prime \prime}\left(q_{1}\right) \times \prod_{\alpha=1}^{r}\left(\mathscr{B}\left(p_{\alpha}\right)\right)^{2} \times \prod_{\beta=1}^{(s-1) / 2}\left(\mathscr{B}\left(q_{2 \beta}\right) \times \mathscr{B}\left(q_{2 \beta+1}\right)\right)^{2} . \\
& \varepsilon=0, s \text { even, } s \neq 0: \\
& \mathscr{D}=\left(\mathscr{B}^{\prime}\left(q_{1}\right) \times \mathscr{B}\left(q_{2}\right)\right) \times \prod_{\alpha=1}^{r}\left(\mathscr{B}\left(p_{\alpha}\right)\right)^{2} \times \prod_{\beta=2}^{s / 2}\left(\mathscr{B}\left(q_{2 \beta-1}\right) \times \mathscr{B}\left(q_{2 \beta}\right)\right)^{2} . \\
& \varepsilon=1, s \text { odd: } \\
& \mathscr{D}=\left(\mathscr{B}^{\prime}(2) \times \mathscr{B}\left(q_{1}\right)\right) \times \prod_{\alpha=r}^{r}\left(\mathscr{B}\left(p_{\alpha}\right)\right)^{2} \times \prod_{\beta=1}^{(s-1) / 2}\left(\mathscr{B}\left(q_{2 \beta}\right) \times \mathscr{B}\left(q_{2 \beta+1}\right)\right)^{2} . \\
& \varepsilon=1, s \text { even, } s \neq 0: \\
& \mathscr{D}=\mathscr{B}(2) \times \prod_{\alpha=r}^{r}\left(\mathscr{B}\left(p_{\alpha}\right)\right)^{2} \times \prod_{\beta=1}^{s / 2}\left(\mathscr{B}\left(q_{2 \beta-1}\right) \times \mathscr{B}\left(q_{2 \beta}\right)\right)^{2} . \\
& \varepsilon=0, s=0: \\
& \mathscr{D}=\mathscr{B}\left(p_{1}\right) \times \prod_{\alpha=2}^{r}\left(\mathscr{B}\left(p_{\alpha}\right)\right)^{2} . \\
& \varepsilon=1, s=0: \\
& \mathscr{D}=\mathscr{B}(2) \times \prod_{\alpha=1}^{r}\left(\mathscr{B}\left(p_{\alpha}\right)\right)^{2} .
\end{aligned}
$$

Note that, at each step in the construction of $\mathscr{D}$, Theorem 2.12 or the remark following it is applicable. Furthermore the Azumaya character corresponding to $\mathscr{D}$ takes values in $\mathbb{Q}(\sqrt{m})$. Finally, applying Lemma 2.15 , we see that the corresponding Azumaya algebra has Hasse index 1/2 at all infinite places.

Corollary 2.17. The Schur group conjecture is true for number rings of subcyclotomic number fields.

Proof. This now follows from Theorem 1.5 and Theorem 2.16.

Corollary 2.18. If $K$ is a subcyclotomic number field with number ring $R$, then $\mathrm{S}(R)=C_{2}$ if $K$ has an even number of real places and $\mathrm{S}(R)=0$ otherwise. 
Proof. This follows immediately from the proof of Theorem 1.5 and from Theorem 2.16.

\section{ACKNOWLEDGMENT}

The author wishes to thank Professor F. M. J. Van Oystaeyen for his continuous advice and support.

\section{REFERENCES}

1. S. Amitsur, Finite subgroups of division rings, Trans. Amer. Math. Soc. 80 (1955), 361-386.

2. S. Caenepeel and F. M. J. Van Oystaeyen, A note on generalized Clifford algebras and projective representations, Comm. Algebra, 17 (1989), 93-102.

3. C. W. Curtis and I. Reiner, Methods of representation theory with applications to finite groups and orders 1, Pure Appl. Math., Wiley, New York, 1981.

4. F. De Meyer and E. Ingraham, Separable algebras over commutative rings, Lecture Notes in Math., vol. 181, Springer-Verlag, Berlin, 1978.

5. F. De Meyer and R. Mollin, The Schur group, orders and their applications, Lecture Notes in Math., vol. 1142, Springer-Verlag, Berlin, 1985, pp. 205-209.

6. __ The Schur group of a commutative ring, J. Pure Appl. Algebra 35 (1985), 117-122.

7. L. Dornhoff, Group representation theory, vol. 1, Pure Appl. Math., vol. 7, Dekker, 1971.

8. W. Feit, The computation of Schur indices, Israel J. Math. 46 (1983), 275-300.

9. J. M. Fontaine, Sur les decompositions des algèbres de groupes, Ann. Sci. École Norm. Sup. (4), 4 (1971), 121-180.

10. G. J. Janusz, Simple components of $\mathbb{Q}[\mathrm{Sl}(2, q)]$, Comm. Algebra 1 (1974), 1-22.

11. P. Nelis and F. M. J. Van Oystaeyen, The projective Schur subgroup of the Brauer group and root groups of finite groups, J. Algebra (to appear).

12. P. Nelis, Integral matrices spanned by finite groups, J. Pure Appl. Algebra, submitted.

13. M. Orzech and Ch. Small, The Brauer group of commutative rings, Lecture Notes in Pure and Appl. Math., vol. 11, Dekker, New York, 1975.

14. I. Reiner, Maximal orders L. M. S. Monographs, vol. 5, 1975.

15. C. R. Riehm, Linear and quadratic Schur subgroups, lecture notes, 1988, preprint.

16. _. The linear and quadratic Schur subgroups over the S-integers of a number field, Proc. Amer. Math. Soc. 107 (1989), 83-87.

17. $\ldots$, The Schur subgroup of the Brauer group of cyclotomic rings of integers, Proc. Amer. Math. Soc., 103 (1988), 27-30.

18. J. P. Serre, Représentations linéaires des groupes finis, Collection Méthodes, Hermann Paris, 1971.

19. S. Tanaka, Construction and classification of irreducible representations of special linear group of the second order over a finite field, Osaka J. Math. 4 (1967), 65-84.

20. J. G. Thompson, Finite groups and even lattices, J. Algebra. 38 (1976), 523-524.

21. M. F. Vigneras, Arithmétique des algèbres de quaternions, Lecture Notes in Math., vol. 800, Springer-Verlag, Berlin, 1980.

22. T. Yamada, The Schur subgroup of the Brauer group, Lecture Notes in Math., vol. 397, Springer-Verlag, Berlin, 1974.

UNiVersity of ANTWERP (UIA), Departement Wiskunde, UNiversiteitsPlein 1, B-2610 WILRIJK, BELGIUM 